OPEN ACCESS

Edited by:

Bin Yang,

University of Leicester,

United Kingdom

Reviewed by:

Gang Huang,

The First Affiliated Hospital of Sun

Yat-sen University, China

Helong Dai,

Central South University, China

*Correspondence:

Cheng Yang

esuperyc@163.com

yang.cheng1@zs-hospital.sh.cn

Ruiming Rong

rong.ruiming@zs-hospital.sh.cn

Tongyu Zhu

tyzhu_dr@163.com

tyzhu@fudan.edu.cn

†These authors have contributed equally to this work

Specialty section

This article was submitted to Nephrology,

a section of the journal

Frontiers in Medicine

Received: 04 September 2021 Accepted: 10 January 2022 Published: 10 February 2022

Citation:

Wang J, Li J, Chen Z, Xu M, Yang C,

Rong $R$ and Zhu $T$ (2022) A

Nomogram for Predicting BK Virus

Activation in Kidney Transplantation

Recipients Using Clinical Risk Factors.

Front. Med. 9:770699.

doi: 10.3389/fmed.2022.770699

\section{A Nomogram for Predicting BK Virus Activation in Kidney Transplantation Recipients Using Clinical Risk Factors}

\author{
Jiyan Wang ${ }^{1,2 \dagger}$, Jiawei $\mathrm{Li}^{1,2 \dagger}$, Zhongli Chen ${ }^{3 \dagger}$, Ming Xu ${ }^{1,2}$, Cheng Yang ${ }^{1,2,4 *}$, \\ Ruiming Rong ${ }^{1,2,5 *}$ and Tongyu Zhu ${ }^{1,2 *}$ \\ ${ }^{1}$ Department of Urology, Zhongshan Hospital, Fudan University, Shanghai, China, ${ }^{2}$ Shanghai Key Laboratory of Organ \\ Transplantation, Shanghai, China, ${ }^{3}$ State Key Laboratory of Cardiovascular Disease, Fuwai Hospital, National Center for \\ Cardiovascular Diseases, Chinese Academy of Medical Sciences and Peking Union Medical College, Beijing, China, \\ ${ }^{4}$ Zhangjiang Institute of Fudan University, Shanghai, China, ${ }^{5}$ Department of Transfusion, Zhongshan Hospital, Fudan \\ University, Shanghai, China
}

BK virus is a common opportunistic viral infection that could cause BK virus-associated nephropathy in renal transplant recipients. Thus, we retrospectively analyzed clinical and laboratory data associated with a higher risk of BK virus activation from 195 renal transplant recipients by the multivariate logistic regression analysis and performed the external validation. Results showed that patients with BK virus active infection were associated with a deceased donor, had lower direct bilirubin levels, a higher proportion of albumin in serum protein electrophoresis, and lower red blood cells and neutrophil counts. The multivariate logistic regression analyses revealed that the living donor, direct bilirubin, and neutrophil counts were significantly associated with BK virus activation. The logistic regression model displayed a modest discriminability with the area under the receiver operating characteristic curve of 0.689 (95\% Cl: $0.607-0.771 ; P<0.01$ ) and also demonstrated a good performance in the external validation dataset (the area under the receiver operating characteristic curve was $0.699,95 \% \mathrm{Cl}: 0.5899-0.8081)$. The novel predictive nomogram achieved a good prediction of BK virus activation in kidney transplant recipients.

Keywords: BK virus, kidney transplantation, risk factor, predictive model, nomogram

\section{INTRODUCTION}

$\mathrm{BK}$ virus (BKV) is a common post-transplant opportunistic viral infection that can cause interstitial nephritis and allograft failure in renal transplant recipients (RTRs) $(1,2)$. With the progress of the immunosuppressive regimen, acute rejection incidence decreased. However, viral infections after renal transplantation are still a hurdle, which causes chronic allograft loss. BKV is one member of the polyomavirus family, which was first described in 1971 in the urine of an RTR with ureteric stenosis. BKV has circular nucleic acid and double-stranded DNA (3). The transmission mechanism of $\mathrm{BKV}$ is unclear but supposed through the mouth and respiratory tract (4). 
Primary infection of $\mathrm{BKV}$, such as intermittent asymptomatic viral shedding in the urine, can be detected in healthy individuals with no adverse outcome. However, in the immunosuppressed status, BKV will be reactivated, leading to clinically BKV disease among the renal and hematopoietic stem cell recipients (5). After being activated in the early stage of BK virus-associated nephropathy (BKPyVN), BKV in the urothelium and tubular epithelium will replicate in high levels. Some virus compositions, such as BKV-DNA and decoy cells, can be detected in urine, diagnosed as BKV viruria. As the damage escalates, the BKV virus will invade into circulation through capillary, causing the $\mathrm{BKV}$ viremia. According to previous studies, the prevalence of $\mathrm{BK}$ viruria, $\mathrm{BK}$ viremia, and $\mathrm{BKPyVN}$ range from 10 to $40 \%$ in RTRs, and this progressively affects graft function and increases the risk of graft loss more than 10\% (4, 6-9). Besides, BKV was also associated with de-novo donor-specific antibodies, which were linked to antibody-mediated rejection (10). In addition, the association between BKV and malignancy has attracted more attention. Some kinds of human neoplasms such as urothelial bladder cancer can detect BKV-DNA sequences and $\mathrm{T}$ antigen (11-13).

Detection of BKV-DNA load in urine and plasma by PCR is a common and effective way to diagnose and monitor BKPyVN and monitor BKPyVN at the early stage. Positive BKV-DNA in urine or plasma has a good negative predictive value, but the positive predictive value is unsatisfactory (14). Current investigations showed that RTRs with a BKV-DNA load of more than $1.0 \times 10^{7}$ copies $/ \mathrm{ml}$ in urine or more than $1 \times$ $10^{4}$ copies $/ \mathrm{ml}$ in plasma have a higher risk of developing into BKPyVN (15-17). This state is considered as BKV activation. This DNA level is also found linked to hemorrhagic cystitis (1820). Some researchers suggested that the viral replication capacity is associated with rearranged-noncoding control regions (21). Unfortunately, effective BK virus-specific antiviral therapies are not available. Therefore, early diagnosis of BKV activation and intervention is of great clinical importance $(22,23)$.

In this study, we analyzed the clinical and laboratory data among 195 RTRs to build a predictive model for BKV active replication.

\section{METHODS}

\section{Patients}

In the training cohort, we selected 196 patients undergoing renal transplantation in Zhongshan Hospital, Fudan University from February 2018 to April 2020. Only RTRs who accepted complete follow-up laboratory examinations and BKV-DNA testing in plasma and urine were included. Inclusion criteria were: living or deceased donor kidney transplant and recipient age $\geq 18$ years (24). Exclusion criteria included recipients with primary immune dysfunction, acute and central nervous system diseases,

\footnotetext{
Abbreviations: BKPyVAN, BK virus-associated nephropathy; BKV, BK virus; DB, direct bilirubin; eGFR, estimated glomerular filtration rate; OR, Odds Ratios; PCR, polymerase chain reaction; RBC, red blood cells; ROC, Receiver Operating Characteristic Curve; AUROC, the area under ROC; RTRs, renal transplant recipients; SPE, serum protein electrophoresis.
}

hemolytic anemia, coagulation dysfunction, HIV and other highrisk pathogens infection, pregnant or lactating women, and other conditions that the investigators deemed inappropriate for participation during the study period. After all, the demographic and clinical information data were collected, 176 RTRs were included (Figure 1). All the patients were divided into two groups according to BKV-DNA levels in plasma and urine. The BKV activation group was defined as BKV-DNA $>10^{7}$ copies $/ \mathrm{L}$ in the urine or $>10^{4}$ copies/L in the plasma $(n=42)$. Others were defined as the BKV inactivation group $(n=134)$. The external cohort with 134 RTRs was enrolled from May 2020 to Oct 2021 in Zhongshan Hospital, Fudan University.

This study was approved by the Institutional Review Board (IRB) at Zhongshan Hospital, Fudan University, Shanghai, China (approval. No.: B2021-074R) and registered and recorded at the Human Genetic Resource Administration of China (HGRAC) (reference no.: 2021BAT1438).

\section{Data Collection}

The demographic features (gender and age), disease history (diabetes, coronary heart disease, hypertension, transfusion, malignancy, hyperlipemia, and viral hepatitis history), and transplantation history (source of donor, transplant number, $\mathrm{ABO}$ compatibility, ischemia time, acute rejection or delayed graft function history, induction treatment, and initial immunosuppressive protocol) were collected by two clinicians. The post-transplantation laboratory tests [blood routine included: red blood cells (RBCs) count, platelets count, neutrophils count, lymphocyte count, and monocyte count; liver function including alanine aminotransaminase, aspartate aminotransferase, total bilirubin, and direct bilirubin (DB); renal function included serum creatine, urea nitrogen, estimated glomerular filtration rate (eGFR), and uric acid; the proportion of serum protein electrophoresis (SPE); and urine protein semiquantitative levels] were also collected at the same time of BKV-DNA test.

\section{Statistical Analysis}

For continuous variables, the one fitting normal distribution is expressed as the mean $\pm \mathrm{SD}$, otherwise described as the median with interquartile ranges. For categorical data, the proportions and frequencies are calculated. Continuous and categorical variables were compared using the independent $t$-test or nonparametric and the chi-squared tests, respectively. The obvious abnormal distribution variables were normalized by natural logarithmic transformation. Spearman's correlation analyses were adopted to explore the relationship between variables.

The univariate analysis was performed to screen the potential variables associated with BKV activation. Variables with $P$ value $<0.1$ in the univariate analysis (living-related donor, DB, globulin, SPE-albumin, SPE- $\beta$, RBC, platelet, and neutrophil counts (as a natural logarithm-transformed continuous variable, represented as "ln Neutr")], demographic features, and clinically important factors such as serum creatinine were further included in the multivariate logistic regression analysis (25). Using the forward stepwise method, the final logistic regression model was 


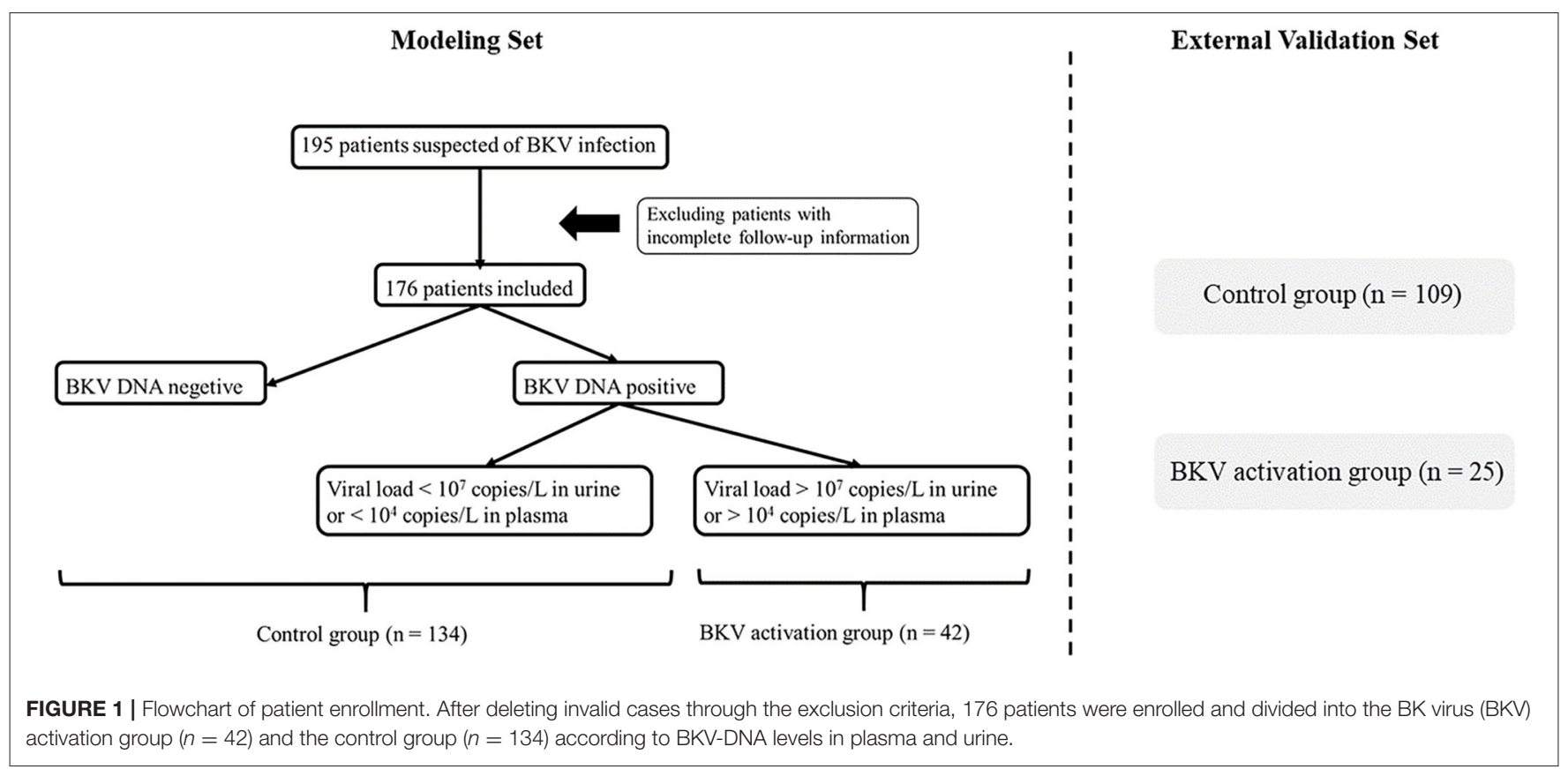

constructed. Odds ratios (ORs) and 95\% CIs were presented. For clinical usage, a nomogram was constructed based on the model. The goodness-of-fit was estimated by the Hosmer-Lemeshow test and discriminability was assessed by the area under the receiver operating characteristic (AUROC) curve.

The performance of the model was also evaluated in the different age and sex groups as well as an external validation cohort. All the statistical analyses were performed using SPSS software, version 20.0 (SPSS Incorporation, Chicago, Illinois, USA) and R software version 3.6.3. Statistical significance was set at two-tailed and $p<0.05$ was considered as statistically significant. All the authors had full access to the data in this study and took responsibility for data integrity and accuracy of data analysis.

\section{RESULTS}

\section{Characteristics of Patients}

The variables included age, gender, the history of diabetes, coronary heart disease, hypertension, transfusion, malignancy, hyperlipemia, viral hepatitis, the information of donor source, transplantation times, ABO compatible, ischemia time, acute rejection or delayed graft function history, induction treatment, initial immunosuppressive protocol (include the combined regimen of tacrolimus, mycophenolic acid, and prednisone, represented as Tac $+\mathrm{MPA}+$ Pred and the combined regimen of cyclosporin A, mycophenolic acid, and prednisone, represented as CsA + MPA + Pred), and BKV-DNA quantitative results in plasma and urine. Other routine follow-up laboratory results were also included: $\mathrm{RBC}$, platelet, neutrophil count, lymphocyte count, monocyte count, total bilirubin, DB, globulin, the proportion of SPE, alanine aminotransferase, aspartate aminotransferase, creatine, urea nitrogen, uric acid, estimated glomerular filtration rate (eGFR), and urine protein semiquantitative levels.

The clinical characteristics are shown in Table 1. There are 65 patients with BKV-DNA positive in urine or plasma and 42 of them were in virus activation according to virus load. When comparing the control group $(n=134)$ and the BKV activation group $(n=42)$, living donor proportion (28.4 vs. $9.5 \%)$, DB $[2.9(1.7,4.0)$ vs. $2.4(1.6,2.8) \mu \mathrm{mol} / \mathrm{l}]$, SPE-Albumin $(61.7 \pm$ 5.0 vs. $63.5 \pm 4.2 \%)$, and $\mathrm{RBC}$ count $\left(3.90 \pm 0.83 \times 10^{12} / \mathrm{L}\right.$ vs. $\left.3.52 \pm 0.72 \times 10^{12} / \mathrm{L}\right)$ showed significant difference. The two groups did not differ with respect to gender, age, the history of diabetes, coronary heart disease, hypertension, transfusion, malignancy, hyperlipemia, viral hepatitis, the information of transplantation times, ABO compatible, ischemia time, acute rejection or delayed graft function history, induction treatment, initial immunosuppressive protocol, the levels of total bilirubin, total protein, globulin, alanine aminotransferase, aspartate aminotransferase, creatine, urea nitrogen, uric acid, eGFR in serum, the proportion of serum $\alpha 1, \alpha 2, \beta, \gamma$ protein in SPE, the count of platelet, neutrophils, lymphocytes, and monocytes in peripheral blood and urine protein semiquantitative levels.

In the external validation cohort, patients $(n=134)$ were among middle-aged ( $46 \pm 12$ years) and $64.9 \%$ were men. A total of 25 patients were divided into the BKV activation group. The clinical characteristics are shown in Supplementary Table S1. Comparing the two groups, neutrophil count $\left[5.3(3.4,7.1) \times 10^{9}\right.$ vs. $\left.4.3(3.2,5.4) \times 10^{9}\right]$ showed a significant statistical difference. The two groups did not differ with respect to gender, age, the history of diabetes, coronary heart disease, hypertension, transfusion, malignancy, hyperlipemia, viral hepatitis, the information of transplantation times, $\mathrm{ABO}$ compatible, ischemia time, acute rejection or delayed graft function history, induction treatment, initial immunosuppressive 
TABLE 1 | Baseline characteristics for patients with or without BKV active replication.

\begin{tabular}{|c|c|c|c|c|}
\hline & $\begin{array}{l}\text { All } \\
(n=176)\end{array}$ & $\begin{array}{l}\text { Inactivated } \\
(n=134)\end{array}$ & $\begin{array}{l}\text { Activated } \\
(n=42)\end{array}$ & P-value \\
\hline Sex & & & & 0.669 \\
\hline Male & $122(69.3)$ & 94 (70.1) & $28(66.7)$ & \\
\hline Female & $54(30.7)$ & $40(29.9)$ & 14 (33.3) & \\
\hline Age & $43.0 \pm 12$ & $42.2 \pm 12.1$ & $45.4 \pm 11.8$ & 0.139 \\
\hline Diabetes & $30(17.0)$ & $24(17.9)$ & $6(14.3)$ & 0.586 \\
\hline Coronary heart disease & $2(1.1)$ & $2(1.5)$ & $0(0)$ & 0.579 \\
\hline Hypertension & $150(85.2)$ & $118(88.1)$ & $41(97.6)$ & 0.126 \\
\hline Transfusion & $19(10.8)$ & $14(10.4)$ & $5(11.9)$ & 1.000 \\
\hline Malignancy & $4(2.3)$ & $3(2.2)$ & $3(7.1)$ & 0.744 \\
\hline Hyperlipemia & $21(11.9)$ & $15(11.2)$ & $6(14.3)$ & 0.590 \\
\hline \multicolumn{5}{|l|}{ Hepatitis } \\
\hline HBV & $26(14.8)$ & $16(11.9)$ & $10(23.8)$ & 0.059 \\
\hline $\mathrm{HCV}$ & $3(1.7)$ & $3(2.2)$ & $0(0)$ & 1.000 \\
\hline First time transplant & $168(95.5)$ & $128(95.5)$ & $40(95.2)$ & 1.000 \\
\hline Donor source & & & & 0.012 \\
\hline Living donor & $42(23.9)$ & $38(28.4)$ & $4(9.5)$ & \\
\hline Deseased donor & $134(76.1)$ & 96 (71.6) & $38(90.5)$ & \\
\hline ABO-compatible & $173(98.3)$ & $132(98.5)$ & 41 (97.6) & 0.699 \\
\hline \multicolumn{5}{|l|}{ Ischemia time } \\
\hline Cold ischemia time (hour) & $5.1(1.0,9.5)$ & $5.0(0.9,9.5)$ & $5.1(0.9,10)$ & 0.863 \\
\hline Warm ischemia time (min) & $2.7(2.0,3.0)$ & $2.6(2.0,3.0)$ & $2.7(2.0,3.0)$ & 0.470 \\
\hline Acute rejection & $2(1.1)$ & 0 & $2(4.8)$ & 0.056 \\
\hline Delayed graft function & $14(8.0)$ & $12(9.0)$ & $2(4.8)$ & 0.583 \\
\hline Intravenous immunogloblin & $2(1.1)$ & $2(1.5)$ & $0(0)$ & 1.000 \\
\hline Induction treatment & & & & 1.000 \\
\hline Antithymocyte globulin & $8(4.5)$ & $6(4.5)$ & $2(4.8)$ & \\
\hline Basiliximab & $168(95.5)$ & $128(95.5)$ & $40(95.2)$ & \\
\hline Initial immunosuppressive protocol & & & & 0.171 \\
\hline Tac+MPA+Pred & $112(63.6)$ & $89(66.4)$ & $23(54.8)$ & \\
\hline CsA+MPA+Pred & $64(36.4)$ & 45 (33.6) & $19(45.2)$ & \\
\hline \multicolumn{5}{|l|}{ Kidney and liver function } \\
\hline Total bilirubin $(\mu \mathrm{mol} / \mathrm{L})$ & $8.0(5.8,10.4)$ & $8.2(5.8,11.3)$ & $7.5(5.7,9.0)$ & 0.139 \\
\hline $\mathrm{DB}(\mu \mathrm{mol} / \mathrm{L})$ & $2.6(1.7,3.5)$ & $2.9(1.7,4.0)$ & $2.4(1.6,2.8)$ & 0.012 \\
\hline Total protein (g/L) & $65 \pm 7$ & $66 \pm 8$ & $64 \pm 6$ & 0.047 \\
\hline Albumin (g/L) & $42 \pm 5$ & $43 \pm 5$ & $42 \pm 3$ & 0.247 \\
\hline Globulin (g/L) & $22 \pm 4$ & $23 \pm 4$ & $22 \pm 4$ & 0.066 \\
\hline Alanine aminotransferase $(\mathrm{U} / L)$ & $15(9,27)$ & $15(9,26)$ & $14(9,29)$ & 0.804 \\
\hline Aspartic aminotransferase (U/L) & $16(13,21)$ & $16(13,21)$ & $15(11,21)$ & 0.465 \\
\hline Creatine $(\mu \mathrm{mol} / \mathrm{L})$ & $152(113,196)$ & $152(113,189)$ & $154(116,272)$ & 0.497 \\
\hline Urea nitrogen (mmol/L) & $9.2(7.2,12.8)$ & $9.2(7.2,12.7)$ & $9.3(7.2,13.8)$ & 0.569 \\
\hline Uric acid ( $\mu \mathrm{mol} / \mathrm{L})$ & $387 \pm 113$ & $390 \pm 115$ & $376 \pm 106$ & 0.480 \\
\hline eGFR (ml/min/1.73m²) & $47 \pm 23$ & $48 \pm 22$ & $43 \pm 24$ & 0.223 \\
\hline \multicolumn{5}{|l|}{ Serum Protein electrophoresis } \\
\hline Albumin (\%) & $62.2 \pm 4.9$ & $61.7 \pm 5.0$ & $63.5 \pm 4.2$ & 0.043 \\
\hline$\alpha 1(\%)$ & $4.8 \pm 1.2$ & $4.8 \pm 1.3$ & $4.6 \pm 1.1$ & 0.524 \\
\hline$\alpha 2(\%)$ & $9.7 \pm 2.3$ & $9.8 \pm 2.3$ & $9.3 \pm 2.0$ & 0.214 \\
\hline$\beta(\%)$ & $10.3 \pm 1.4$ & $10.4 \pm 1.4$ & $9.9 \pm 1.2$ & 0.074 \\
\hline$\gamma(\%)$ & $13.1 \pm 3.3$ & $13.2 \pm 3.3$ & $12.6 \pm 3.3$ & 0.265 \\
\hline \multicolumn{5}{|l|}{ Blood count } \\
\hline $\operatorname{RBC}\left(\times 10^{12} / L\right)$ & $3.81 \pm 0.82$ & $3.90 \pm 0.83$ & $3.52 \pm 0.72$ & 0.008 \\
\hline
\end{tabular}




\begin{tabular}{|c|c|c|c|c|}
\hline & $\begin{array}{l}\text { All } \\
(n=176)\end{array}$ & $\begin{array}{l}\text { Inactivated } \\
(n=134)\end{array}$ & $\begin{array}{l}\text { Activated } \\
(n=42)\end{array}$ & P-value \\
\hline PLT/10 (×109/L) & $19.9 \pm 6.6$ & $20.4 \pm 6.9$ & $18.3 \pm 5.3$ & 0.072 \\
\hline Lymphocyte $\left(\times 10^{9} / \mathrm{L}\right)$ & $1.6 \pm 0.8$ & $1.6 \pm 0.8$ & $1.4 \pm 0.7$ & 0.200 \\
\hline Monocyte $\left(\times 10^{9} / \mathrm{L}\right)$ & $0.58 \pm 0.24$ & $0.58 \pm 0.24$ & $0.59 \pm 0.22$ & 0.940 \\
\hline$( \pm)$ & $36(20.5)$ & 28 (20.9) & $8(19.0)$ & \\
\hline$(+\sim++)$ & $16(9.1)$ & $9(6.7)$ & $7(16.7)$ & \\
\hline$(++\sim+++)$ & $9(5.1)$ & $9(6.7)$ & I & \\
\hline$(+++\sim++++)$ & $3(1.7)$ & $3(2.2)$ & I & \\
\hline
\end{tabular}

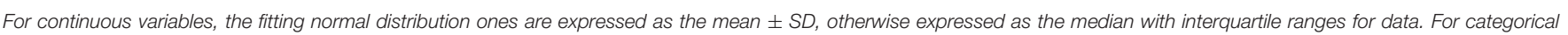
data, the proportions and frequencies are calculated. Continuous and categorical variables were compared using the independent $t$-test or the nonparametric and chi-squared tests. BKV, BK virus; DB, direct bilirubin; Tac, tacrolimus; CSA, cyclosporine A; MPA, mycophenolic acid; Pre, prednisone; eGFR, estimated glomerular filtration rate; RBC, red blood cell.

protocol, the levels of total bilirubin, DB, total protein, albumin, globulin, alanine aminotransferase, aspartate aminotransferase, creatine, urea nitrogen, uric acid, eGFR in serum, the proportion of serum $\alpha 1, \alpha 2, \beta, \gamma$ protein in SPE, the count of RBC, platelet, neutrophils, lymphocytes, and monocytes in peripheral blood and urine protein semiquantitative levels.

\section{Clinical Factors Associated With BKV Activation}

The univariate analyses showed that BKV activation was associated with living donors, DB (as a natural logarithmtransformed continuous variable, represented as "ln DB"), globulin, SPE- $\beta$, RBC, platelet, and neutrophil counts (as a natural logarithm-transformed continuous variable, represented as "ln Neutr") (Table 2). In the multivariate logistic regression analyses, the living donor, DB level, and neutrophil count were selected as significant variables in the model. Specifically, for patients receiving the kidney graft from a living donor, there was a $74 \%$ lower likelihood of having BKV activation status. Among patients in our study, higher levels of DB and neutrophil counts were associated with lower odds of BKV activation.

\section{Evaluation of Model Performance}

The logistic regression model $[\mathrm{P} /(1-\mathrm{P})=\exp [0.745-1.347$ (living donor) -0.691 (ln DB) -0.720 (ln Neutr)] displayed a good fit with a Nagelkerke $\mathrm{R}^{2}$ value of 0.143 and the nonsignificant Hosmer-Lemeshow test. The ROC analysis showed that the AUROC of the model was 0.689 (95\% CI: $0.607-0.771 ; P$ $<0.01$ ) for distinguishing the presence of BKV activation (Figure 2), which was better than the creatine or eGFR. To test the robustness of the model, we applied the model to the different age, sex, or eGFR subgroups and an independent cohort. Notably, the model demonstrated higher discriminability in female (AUROC, 0.721 vs. male 0.674 ) and younger patients (AUROC, 0.720 vs. male 0.664) (Figure 3), while its AUROC was roughly equal between the eGFR $\geq 45 \mathrm{ml} / \mathrm{min} / 1.73 \mathrm{~m}^{2}$ and $<45 \mathrm{ml} / \mathrm{min} / 1.73 \mathrm{~m}^{2}$ groups. Additionally, the model retained modest discrimination in the external validation dataset, although the AUROC slightly decreased (0.699, 95\% CI: 0.58990.8081) (Figure 4).

\section{Predictive Nomogram for the Probability of BKV Reactivation}

On the basis of the multivariate logistic regression model, a nomogram was constructed for predicting BKV activation (Figure 5). A total score was calculated using donor source, ln DB and ln Neutr. The value of each of these variables on the corresponding can match a number to a score on the point scale axis. A sum of all the variable scores could draw a line to the total point axis and acquire the probability of BKV activation.

\section{DISCUSSION}

$\mathrm{BK}$ virus infection is a common post-transplant opportunistic infection. The relationship between BKV and renal allograft dysfunction is still unclear and the effective management strategies remain to warrant further investigation. In the view of the facts that are more relevant between BKV activation and graft loss and the few effective BK virus-specific treatment, identifying BKV activation patients as early as possible to increase posttransplant surveillance and promptly adjust treatments are of greater clinical value. In this article, we compared the clinical data and laboratory results among RTRs to build a prediction model for BKV active replication.

Previous studies had come up with many but controversial risk factors of BKV infection, including age, gender, human leukocyte antigen (HLA) mismatches, deceased donor transplants, duration of cold ischemic time, body mass index, and types of immunosuppressive drugs (26-28). All of these have yet to be widespread corroborated. In a multi-center retrospective study including 21,575 mate kidney transplant pairs, age $<18$ or $\geq 60$ years, male sex, depleting antibody, HLA mismatch $\geq 4$ were identified as the risk factors of BKV 
TABLE 2 | The multivariate logistic regression models for renal transplant recipients (RTRs) with BKV activation.

\begin{tabular}{lcccc}
\hline & \multicolumn{2}{c}{ Univariate analysis } & & \multicolumn{2}{c}{ Multivariable analysis } \\
\cline { 2 - 4 } & OR & P-value & OR & P-value \\
\hline Living related donor & 0.266 & 0.018 & 0.260 & 0.019 \\
Ln DB & 0.515 & 0.034 & 0.501 & - \\
Globulin & 0.923 & 0.068 & - & - \\
SPE-Albumin & 1.086 & 0.046 & - & - \\
SPE- $\beta$ & 0.784 & 0.076 & - & - \\
RBC & 0.563 & 0.010 & - & - \\
Platelet & 0.995 & 0.075 & 0.487 & 0.053 \\
Ln Neutr & 0.492 & 0.035 & & -
\end{tabular}

Age, gender, living-related donor, In DB, globulin, serum protein electrophoresis (SPE)-Albumin, SPE- $\beta$, red blood cell (RBC) count, platelet count, and In Neutr were included.

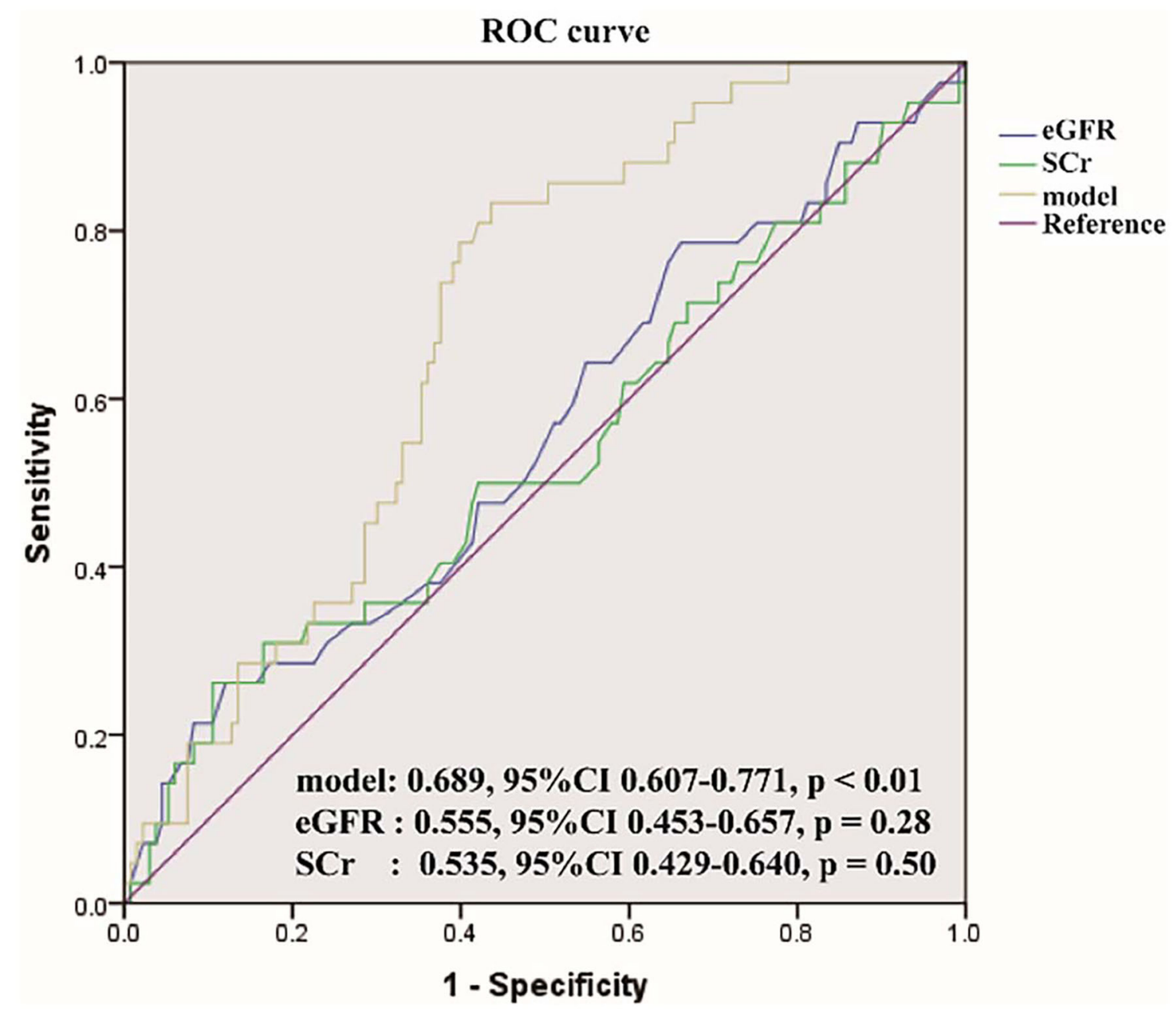

FIGURE 2 | The receiver operating characteristic (ROC) curve of the model compared with serum creatinine (SCr) and estimated glomerular filtration rate (eGFR). The area under the ROC (AUROC) of the three models was $0.689,0.555,0.535$, respectively, indicating that model has better predictive power.

(27). The results showed that age, gender, HLA mismatches, acute rejection, and the use of depleting antibody induction of recipients were associated with a higher odd value of BKV treatment. Another retrospective analysis for identification of BKV infection among living-donor RTRs showed that tacrolimus level and decreased lymphocyte percentage might be the risk 
A

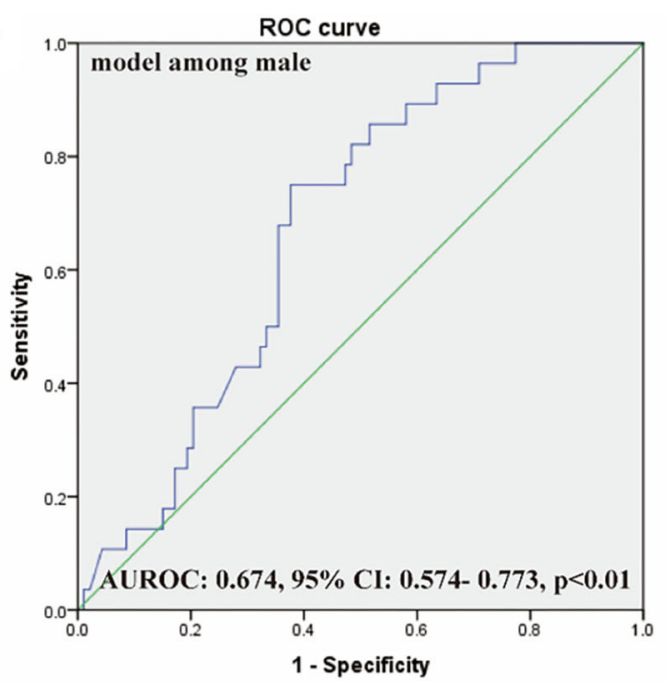

C

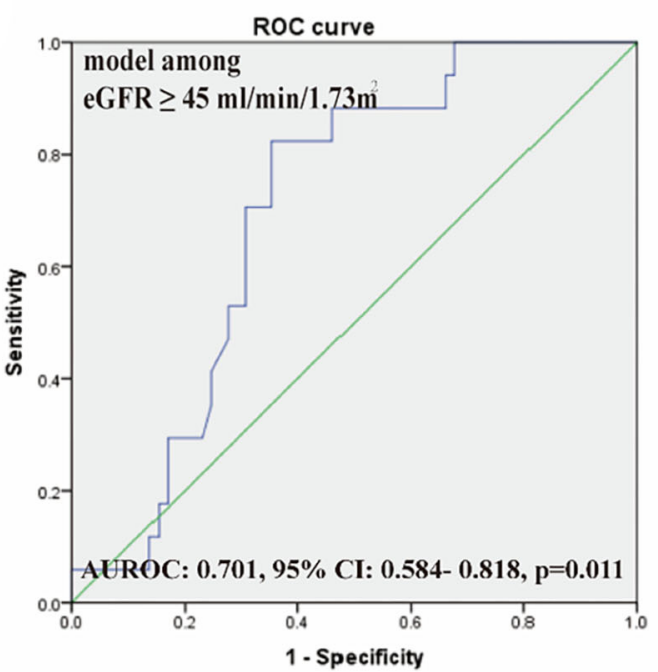

E

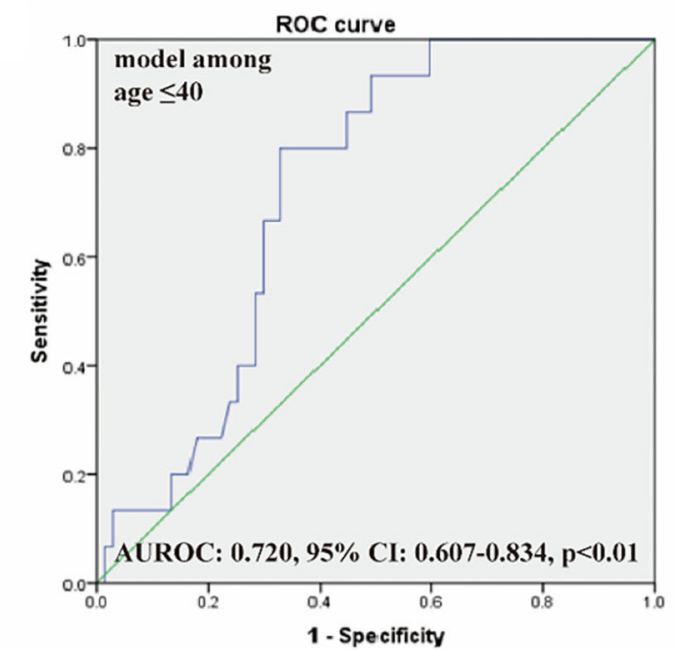

B

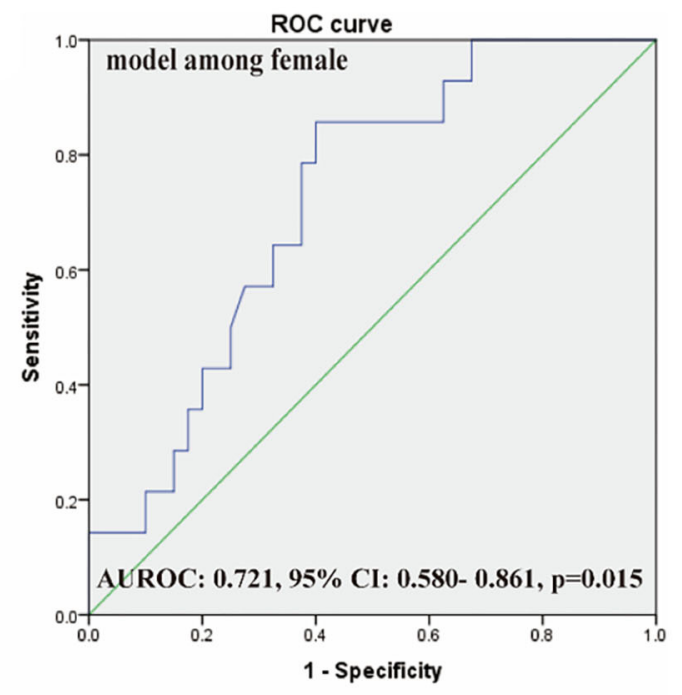

D

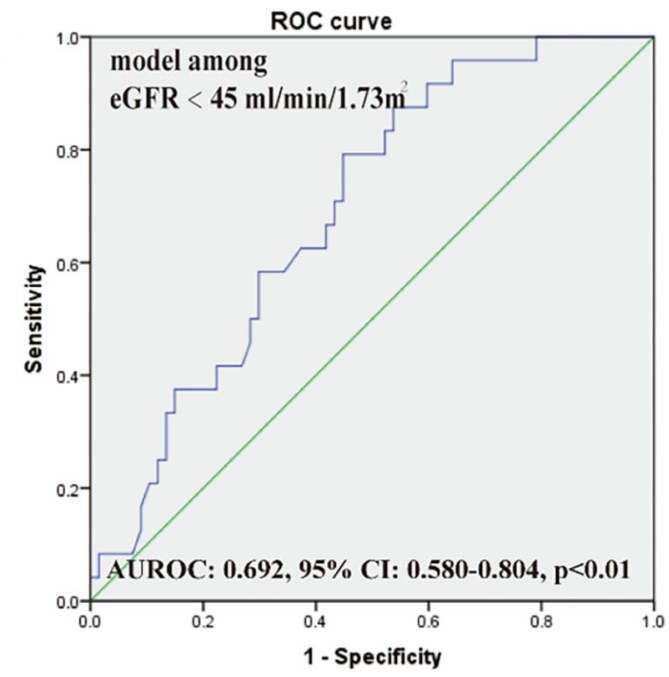

F

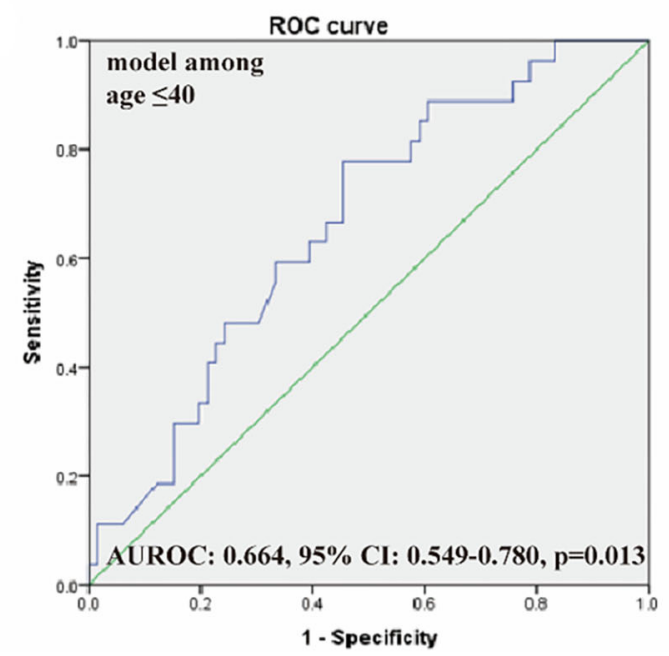

FIGURE 3 | The ROC of our model among male (A) and female patients (B), eGFR $\geq 45 \mathrm{ml} / \mathrm{min} / 1.73 \mathrm{~m}^{2}$ (C) and $<45 \mathrm{ml} / \mathrm{min} / 1.73 \mathrm{~m}{ }^{2}$ (D), age $\leq 40$ years (E) and > 40 years $\mathbf{( F )}$. AUROC, the area under the receiver operating characteristic curve. 


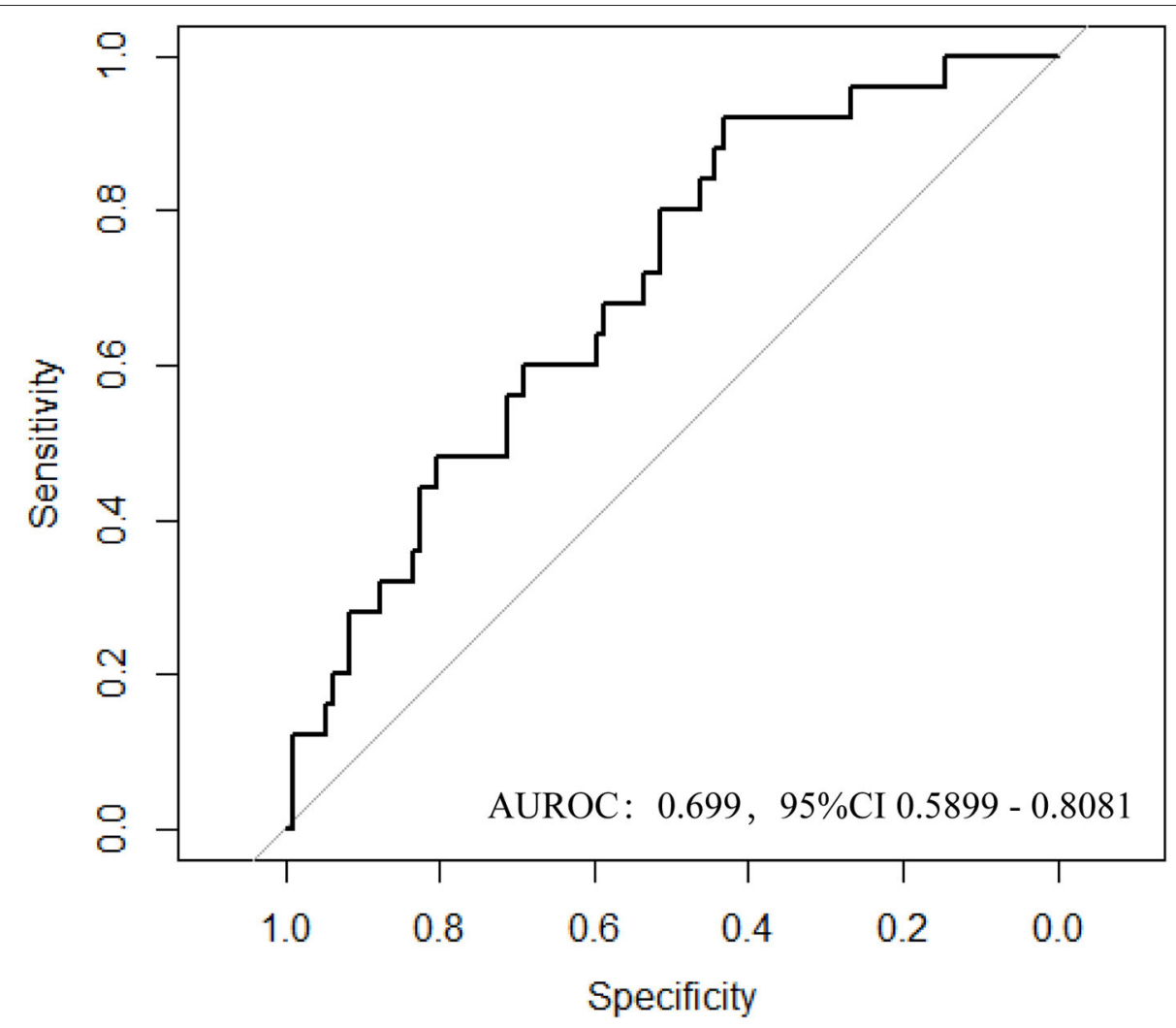

FIGURE 4 | Discriminatory performance in external datasets for adverse BKV infection status.

factors (29). The results vary from research to research, which may be related to the different clinical manifestations between BKV infection and damage.

In our study, an effective predictive model for identifying BKV activation was established by the multivariate logistic regression analyses. In this model, the living donor, tDB level, and neutrophil count were found that have significant differences for predicting the presence of BKV activation. In verifying the model performance, the ROC and the AUROC of the model showed better performance than routine renal function index [included serum creatinine (SCr) and eGFR]. Also, after comparing the ROC of our model among male and female patients, eGFR $\geq 45 \mathrm{ml} / \mathrm{min} / 1.73 \mathrm{~m}^{2}$ and $<45 \mathrm{ml} / \mathrm{min} / 1.73 \mathrm{~m}^{2}$, and age $\leq$ 40 years and $>40$ years, this model showed more power in female and younger patients. For visualization, a nomogram was constructed by incorporating the three significant factors. In further validating the model efficiency, the predictive ability of the model in external validation shown as the ROC still had a certain power.

The common diagnostic measures of BKV include seeking decoy cells in urinary sediment, test virus DNA in urine or plasma by PCR, or graft biopsy. All the measurements have different merits and demerits. The sensitivity of urinary sediment cytology is poor and the positive results often along with the high level of virus replication miss the early. The biopsy is a gold standard for diagnosis of BKPyVN by immunohistochemistry staining SV40 and LT antigen, but it is also an invasive detection and possible to be false positive due to the bias of puncture position. Detection of the viral load in urine and plasma by PCR is an effective way to monitor diseases in the early stage. The levels of BKV-DNA in urine and plasma are highly correlated with BKPyVN. However, positive BKV-DNA in urine or plasma has a good negative predictive value, but an unsatisfactory positive predictive value (14). It is highly sensitive for reminding active viral replication but not for BKPyVN. Current foreign and domestic investigations showed that people whose BKVDNA load is more than $1.0 \times 107$ copies/ml in urine and more than $1 \times 104$ copies/ml in plasma have a higher risk of developing into BKPyVN and need timely intervention (15-17). Meanwhile, there are no effective BK virus-specific antiviral therapies available $(22,23)$. The mainstay for managing reactivation still relies on regular screening accompanied by a reduction of immunosuppressant and adding antiviral drugs, if the former is in vain (30). However, this approach proves effective only in $50-80 \%$ of cases and does not eliminate the potential evolution to BKPyVN (30). Therefore, identifying this kind of patient as early as possible and timely intervention to improve patients' prognosis are of great significance.

The source of the donor was screened as an independent protective factor of BKV activation, which means that RTRs whose graft from living donors are less likely to BKV reactivation. This is consistent with some previous studies. A systematic 


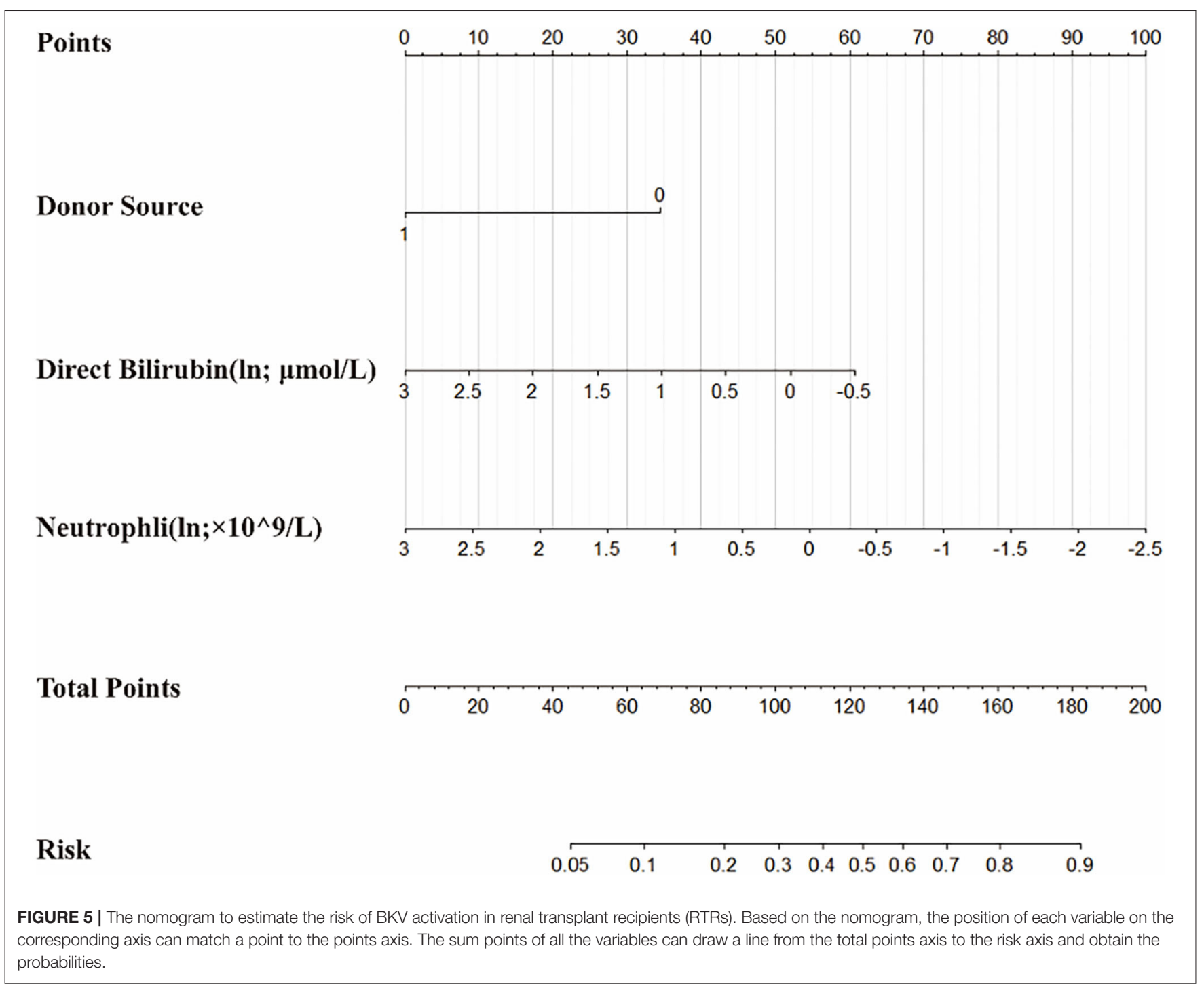

review and meta-analysis of risk factors for BKV viremia and BKPyVAN showed that deceased donor is one of the risk factors (31). In the included multivariate analyses studies, the whole six articles included showed strong associations between $\mathrm{BKPyV}$ viremia and deceased donor condition and the OR value of two of them has statistical differences $(32,33)$. However, there is no clear theoretical basis supporting the correlation between the source of graft and BKV activation. Clinical data seem to only provide this phenomenon but a clue. It can be supposed that the virus activation may not only relate to the recipient condition but also the donor condition.

As a normal marker responding to hepatocyte metabolism, most of DB is derived from the breakdown of hemoglobin ( $\mathrm{Hb})$ in erythrocytes and formulated in the liver (34). Even though a little study of BKV and DB can be retrieved, serum DB levels have been always attended in the clinical studies of other virusassociated diseases. For example, a retrospective study of patients with COVID-19 in Guangzhou found that DB was one of the independent risk factors for the occurrence of critical illness (35). In this study, serum DB levels seem like a predictor factor. The antiviral effect of DB has little clue in current literature, but it was mentioned in hepatitis A virus (HAV) infection. $\mathrm{DB}$ may differentially regulate $\mathrm{CD} 4+\mathrm{T}$ lymphocytes and $\mathrm{T}$ regulatory cells functions by modulating intracellular pathways and cellular receptor expression to affect T-cell function during HAV infection (36). Another study indicated that DB may affect cytokine profiles in HAV infection by modulating signal transducers and activators of transcription proteins acting as a potential immunomodulator (37). Moreover, its protective function may be associated with antioxidation. Some studies suggested that bilirubin, at micromolar concentrations in vitro, efficiently scavenges peroxyl radicals generated chemically in either homogeneous solution or multilamellar liposomes (38). There are other studies in hepatitis virus that found the antioxidant properties and immunomodulation function of DB $(37,39)$. Therefore, combining the basis that BKV reactivation 
and causing related diseases are associated with T lymphocytes, $\mathrm{DB}$ may also play an antiviral effect in BKV activation by modulating $\mathrm{T}$ lymphocytes function.

Neutrophils in peripheral blood are the main components of white blood cells and a common indicator, which can generally reflect body immunologic function. BKV infection has been associated with hemorrhagic cystitis in recipients of hematopoietic stem cell transplants, suggesting that reactivation of this infection might have some relationship with diminished immune surveillance (15). Previous studies strongly supported the occurrence of BKV-associated graft loss or cancer associated with $\mathrm{BKV}$ reactivation due to the low immunity $(4,9)$. In our study, the results that neutrophil count was regarded as a protective factor in BKV activation can be simply explained as mapping the capacity for resisting BKV reactivation. Otherwise, many studies pointed out the correlation between lymphocytes count and BKV, even though the lymphocytes count did not perform obvious results in our analyses. The lymphocyte count was reportedly lower in patients with BKV viremia than in patients with no viremia $(40,41)$. It is unknown whether different parts of leukocytes play a respective role in BKV infection and how various phenotypes lymphocytes regulate the BKV reactivation pathological process.

In our result, the AUROC is different between men and women. One of the reasons may be the original different proportions of kidney transplant recipients between men and women whether of BKV activation. The number of included female cases was less than male in the same period. The statistical result reflected that the power in women was not necessarily accurate. Another reason may be the genetic heterogeneity between the $\mathrm{X}$ and $\mathrm{Y}$ chromosomes leading to sex differences persisting throughout the whole body and life (42). The different power between men and women is possibly objective existence. Further research including more cases in multicenter is needed to explore the model power between both the gender and possible mechanism if the difference exists.

There are some aspects of this study that should be noted as limitations. Our model was a single-center study for 2 years so the limited number of cases included impact on the model power. In addition, the results of performance evaluation showed that the prediction ability of the model is superior to normal renal function but is not excellent. The reason may go back to the included variable, which suggested that high specificity and correlation indexes are waiting for application into clinical practice.

\section{CONCLUSION}

This study suggested several clinical factors associated with BKV activation after renal transplantation and we built a model for identifying BKV status. The potential clinical implications for taking advantage of routine follow-up laboratory examines to monitor and predict BKV infection and activation still need to go further.

\section{DATA AVAILABILITY STATEMENT}

The datasets presented in this study can be found in online repositories. The names of the repository/repositories and accession number(s) can be found below: the Human Genetic Resource Administration of China, https://fuwu.most.gov.cn/, 2021BAT1438.

\section{ETHICS STATEMENT}

This study was approved by the Institutional Review Board (IRB) at Zhongshan Hospital, Fudan University, Shanghai, China (Approval. No.: B2021-074R) and registered and recorded at the Human Genetic Resource Administration of China (HGRAC) (Reference No.: 2021BAT1438).

\section{AUTHOR CONTRIBUTIONS}

$\mathrm{TZ}$, CY, and RR contributed to the conceptualization, methodology, funding acquisition, and project administration. JW, JL, and ZC contributed to the formal analysis, data curation, investigation, and writing review and editing. JW and JL contributed to the writing the original draft preparation. MX contributed to the supervision. All the authors have read and agreed to the published version of the manuscript.

\section{FUNDING}

This study was supported by the National Key R\&D Program of China (2018YFA0107501 to RR, 2018YFA0107502 to CY), the National Natural Science Foundation of China (81770746 to CY, 81770747 and 81970646 to RR), the Shanghai Rising Star Program (19QA1406300 to CY), the Medical and Health Talents Training Plan for the Excellent Youth of Shanghai Municipal (2018YQ50 to CY), the 2019 Shanghai Youth Talent Development Program (to CY), the Science and Technology Commission of Shanghai Municipality (16431902300 to TZ), and the Zhongshan Hospital of China (SYS-054 to TZ).

\section{ACKNOWLEDGMENTS}

We are grateful for the contribution of the study personnel from the Departments of Urology, Laboratory in Zhongshan Hospital, and Shanghai Public Health Clinical Center for persistent contribution to BKV-DNA level tests.

\section{SUPPLEMENTARY MATERIAL}

The Supplementary Material for this article can be found online at: https://www.frontiersin.org/articles/10.3389/fmed. 2022.770699/full\#supplementary-material

Supplementary Table S1 | The clinical characteristics of external validation patients. 


\section{REFERENCES}

1. Randhawa PS, Finkelstein S, Scantlebury V, Shapiro R, Vivas C, Jordan $M$, et al. Human polyoma virus-associated interstitial nephritis in the allograft kidney. Transplantation. (1999) 67:1039. doi: 10.1097/00007890-199901150-00018

2. Purighalla R., Shapiro R, McCauley J, Randhawa P. BK virus infection in a kidney allograft diagnosed by needle biopsy. Am J Kidney Dis. (1995) 26:671-3. doi: 10.1016/0272-6386(95)90608-8

3. Gardner SD, Field AM, Coleman DV, Hulme B. New human papovavirus (B.K.) isolated from urine after renal transplantation. Lancet. (1971) 1:12537. doi: 10.1016/S0140-6736(71)91776-4

4. Hirsch HH, Randhawa P. BK polyomavirus in solid organ transplantation. Am J Transplant. (2013) 179-88. doi: 10.1111/ajt.12110

5. Egli A, Infanti L, Dumoulin A, Buser A, Samaridis J, Stebler C, Gosert R, Hirsch HH. Prevalence of polyomavirus BK and JC infection and replication in 400 healthy blood donors. J Infect Dis. (2009) 199:83746. doi: 10.1086/597126

6. Muñoz P, Fogeda M, Bouza E, Verde E, Palomo J, Bañares R. Prevalence of BK virus replication among recipients of solid organ transplants. Clin Infect Dis. (2005) 41:1720-5. doi: 10.1086/498118

7. Hirsch HH, Brennan DC, Drachenberg CB, Ginevri F, Gordon J, Limaye AP, et al. Polyomavirus-associated nephropathy in renal transplantation: interdisciplinary analyses and recommendations. Transplantation. (2005) 79:1277-86. doi: 10.1097/01.TP.0000156165.83160.09

8. Bohl DL, Brennan DC. BK virus nephropathy and kidney transplantation. Clin J Am Soc Nephrol. (2007) 2:S36-46. doi: 10.2215/CJN.00920207

9. Lamarche C, Orio J, Collette S, Senécal L, Hébert MJ, Renoult É, et al. BK Polyomavirus and the Transplanted Kidney: Immunopathology and Therapeutic Approaches. Transplantation. (2016) 100:2276-87. doi: 10.1097/TP.0000000000001333

10. Sawinski D, Forde KA, Trofe-Clark J, Patel P, Olivera B, Goral s, Bloom RD. Persistent BK viremia does not increase intermediate-term graft loss but is associated with de novo donor-specific antibodies. J Am Soc Nephrol. (2015) 26:966-75. doi: 10.1681/ASN.2014010119

11. Llewellyn MA, Gordon NS, Abbotts B, James ND, Zeegers MP, Cheng $\mathrm{KK}$, et al. Defining the frequency of human papillomavirus and polyomavirus infection in urothelial bladder tumours. Sci Rep. (2018) 8:11290. doi: 10.1038/s41598-018-29438-y

12. Gupta G, Kuppachi S, Kalil RS, Buck CB, Lynch CF, Engels EA. Treatment for presumed BK polyomavirus nephropathy and risk of urinary tract cancers among kidney transplant recipients in the United States. Am J Transplant. (2018) 18:245-252. doi: 10.1111/ajt.14530

13. Papadimitriou JC, Randhawa P, Rinaldo CH, Drachenberg CB, Alexiev B, Hirsch $\mathrm{HH}$. BK polyomavirus infection and renourinary tumorigenesis. Am J Transplant. (2016) 16:398-406. doi: 10.1111/ajt.13550

14. Hirsch HH, Steiger J. Polyomavirus BK. Lancet Infect Dis. (2003) 3:61123. doi: 10.1016/S1473-3099(03)00770-9

15. Ambalathingal GR, Francis RS, Smyth MJ, Smith C, Khanna R. BK polyomavirus: clinical aspects, immune regulation, and emerging therapies. Clin Microbiol Rev. (2017) 30:503-28. doi: 10.1128/CMR.00074-16

16. Marinic K, Sinchi J, Gómez M, Díaz R, Grillo S, Habegger-de Sorrentino A. Monitoring of $\mathrm{BK}$ virus in transplant patients of the renal unit of the Perrando Hospital, Chaco, Argentina. Nefrologia. (2014) 34:799800. doi: 10.3265/Nefrologia.pre2014.Jul.12657

17. Costa C, Bergallo M, Astegiano S, Terlizzi ME, Sidoti F, Segoloni GP, Cavallo R. Monitoring of BK virus replication in the first year following renal transplantation. Nephrol Dial Transplant. (2008) 23:33336. doi: $10.1093 / \mathrm{ndt} / \mathrm{gfn} 289$

18. Lee YJ, Zheng J, Kolitsopoulos Y, Chung D, Amigues I, Son T, et al. Relationship of BK polyoma virus (BKV) in the urine with hemorrhagic cystitis and renal function in recipients of $\mathrm{T}$ Cell-depleted peripheral blood and cord blood stem cell transplantations. Biol Blood Marrow Transplant. (2014) 20:1204-10. doi: 10.1016/j.bbmt.2014.04.017

19. Cesaro S, Tridello G, Pillon M, Calore E, Abate D, Tumino M, et al. A prospective study on the predictive value of plasma BK virus-DNA load for hemorrhagic cystitis in pediatric patients after stem cell transplantation. $J$ Pediatric Infect Dis Soc. (2015) 4:134-42. doi: 10.1093/jpids/piu043
20. Jaiswal SR, Singhal P, Thatai A, Bhagwati G, Aiyer HM, Chakrabarti A, Chakrabarti S. Impact of extended infusional mesna prophylaxis on the incidence of BK viruria and hemorrhagic cystitis following post-transplantation cyclophosphamide and CTLA4Ig-based haploidentical transplantation. Ann Hematol. (2020) 99:839-45. doi: 10.1007/s00277-020-03930-w

21. Gosert R, Rinaldo CH, Funk GA, Egli A, Ramos E, Drachenberg CB, et al. Polyomavirus $\mathrm{BK}$ with rearranged noncoding control region emerge in vivo in renal transplant patients and increase viral replication and cytopathology. $J$ Exp Med. (2008) 205:841-52. doi: 10.1084/jem.20072097

22. Sawinski D, Trofe-Clark J. BK Virus Nephropathy. Clin J Am Soc Nephrol. (2018) 13:1893-6. doi: 10.2215/CJN.04080318

23. Barth H, Solis M, Lepiller Q, Sueur C, Soulier E, Caillard S, et al. 45 years after the discovery of human polyomaviruses BK and JC: Time to speed up the understanding of associated diseases and treatment approaches. Crit Rev Microbiol. (2017) 43:178-95. doi: 10.1080/1040841X.2016.1189873

24. Favi E, Puliatti C, Sivaprakasam R, Ferraresso M, Ambrogi F, Delbue $\mathrm{S}$, et al. Incidence, risk factors, and outcome of $\mathrm{BK}$ polyomavirus infection after kidney transplantation. World J Clin Cases. (2019) 7:27090. doi: 10.12998/wjcc.v7.i3.270

25. Muñoz-Gallego I, Moral N, Pascual C, Alonso Y, Folgueira L. BK virus viral load: analysis of the requests received by the microbiology laboratory and clinical involvement of the issued results. Eur J Clin Microbiol Infect Dis. (2019) 38:1969-73. doi: 10.1007/s10096-019-03632-w

26. Chong S, Antoni M, Macdonald A, Reeves M, Harber M, Magee CN. BK virus: current understanding of pathogenicity and clinical disease in transplantation. Rev Med Virol. (2019) 29:e2044. doi: 10.1002/rmv.2044

27. Thangaraju S, Gill J, Wright A, Dong J, Rose C, Gill J. Risk factors for BK polyoma virus treatment and association of treatment with kidney transplant failure: insights from a paired kidney analysis. Transplantation. (2016) 100:854-61. doi: 10.1097/TP.0000000000000890

28. Thölking G, Schmidt C, Koch R, Schuette-Nuetgen K, Pabst D, Wolters H, et al.. Influence of tacrolimus metabolism rate on BKV infection after kidney transplantation. Sci Rep. (2016) 6:32273. doi: 10.1038/srep32273

29. Li P, Cheng D, Wen J, Xie K, Li X, Ni X, et al. Risk factors for BK virus infection in living-donor renal transplant recipients: a single-center study from China. Ren Fail. (2018) 40:442-6. doi: 10.1080/0886022X.2018.1489843

30. Johnston O, Jaswal D, Gill JS, Doucette S, Fergusson DA, Knoll GA. Treatment of polyomavirus infection in kidney transplant recipients: a systematic review. Transplantation. (2010) 89:1057-70. doi: 10.1097/TP.0b013e3181d0e15e

31. Demey B, Tinez C, François C, Helle F, Choukroun G, Duverlie G, et al. Risk factors for BK virus viremia and nephropathy after kidney transplantation: A systematic review. J Clin Virol. (2018) 109:6-12. doi: 10.1016/j.jcv.2018.10.002

32. Knight RJ, Gaber LW, Patel SJ, DeVos JM, Moore LW, Gaber AO. Screening for BK viremia reduces but does not eliminate the risk of BK nephropathy: a single-center retrospective analysis. Transplantation. (2013) 95:949-54. doi: 10.1097/TP.0b013e31828423cd

33. Dogan SE, Celebi ZK, Akturk S, Kutlay S, Tuzuner A, Keven K, Sengul S. Prevalence and risk factors of BK viremia in patients with kidney transplantation: a single-center experience from Turkey. Transplant Proc. (2017) 49:532-6. doi: 10.1016/j.transproceed.2017.01.009

34. Ngashangva L, Bachu V, Goswami P. Development of new methods for determination of bilirubin. J Pharm Biomed Anal. (2019) 162: 27285. doi: 10.1016/j.jpba.2018.09.034

35. Liang W, Liang H, Ou L, Chen B, Chen A, Li C, et al. Development and validation of a clinical risk score to predict the occurrence of critical illness in hospitalized patients with COVID-19. JAMA Intern Med. (2020) 180:10811089. doi: 10.1001/jamainternmed.2020.2033

36. Corral-Jara KF, Trujillo-Ochoa JL, Realpe M, Panduro A, Gómez-Leyva JF, Rosenstein Y, et al. Conjugated bilirubin differentially regulates $\mathrm{CD} 4+\mathrm{T}$ effector cells and $t$ regulatory cell function through outside-in and insideout mechanisms: the effects of HAV cell surface receptor and intracellular signaling. Mediators Inflamm. (2016) 1759027. doi: 10.1155/2016/1759027

37. Castro-García FP, Corral-Jara KF, Escobedo-Melendez G, SandovalHernandez MA, Rosenstein Y, Roman S, et al. Conjugated bilirubin affects cytokine profiles in hepatitis A virus infection by modulating function of signal transducer and activator of transcription factors. Immunology. (2014) 143:578-87. doi: 10.1111/imm.12336 
38. Stocker R, Yamamoto Y, McDonagh AF, Glazer AN, Ames BN. Bilirubin is an antioxidant of possible physiological importance. Science. (1987) 235:10436. doi: 10.1126/science.3029864

39. Liu Y, Zhu P, Wang W, Tan X, Liu C, Chen Y, et al. Mucosalassociated invariant $\mathrm{T}$ cell dysregulation correlates with conjugated bilirubin level in chronic HBV infection. Hepatology. (2021) 73:16711687. doi: 10.1002/hep.31602

40. Drew RJ, Walsh A, Ní Laoi B, Conneally E, Crowley B. BK virus (BKV) plasma dynamics in patients with BKV-associated hemorrhagic cystitis following allogeneic stem cell transplantation. Transpl Infect Dis. (2013) 15:27682. doi: 10.1111/tid.12066

41. Velioglu A, Aksu B, Asicioglu E, Arikan H, Tinay I, Yardimci $\mathrm{S}$, et al. Association of BK virus titers with lymphocyte count in renal transplant patients. Transplant Proc. (2015) 47:14214. doi: 10.1016/j.transproceed.2015.04.042

42. Mauvais-Jarvis F, Bairey Merz N, Barnes PJ, Brinton RD, Carrero JJ, DeMeo DL, et al. Sex and gender: modifiers of health, disease, and medicine. Lancet. (2020) 396:565-82. doi: 10.1016/S0140-6736(20)31561-0
Conflict of Interest: The authors declare that the research was conducted in the absence of any commercial or financial relationships that could be construed as a potential conflict of interest.

Publisher's Note: All claims expressed in this article are solely those of the authors and do not necessarily represent those of their affiliated organizations, or those of the publisher, the editors and the reviewers. Any product that may be evaluated in this article, or claim that may be made by its manufacturer, is not guaranteed or endorsed by the publisher.

Copyright (C) 2022 Wang, Li, Chen, Xu, Yang, Rong and Zhu. This is an open-access article distributed under the terms of the Creative Commons Attribution License (CC $B Y)$. The use, distribution or reproduction in other forums is permitted, provided the original author(s) and the copyright owner(s) are credited and that the original publication in this journal is cited, in accordance with accepted academic practice. No use, distribution or reproduction is permitted which does not comply with these terms. 